\title{
UBE2O promotes the proliferation, EMT and stemness properties of breast cancer cells through the UBE2O/AMPKa2/mTORC1-MYC positive feedback loop
}

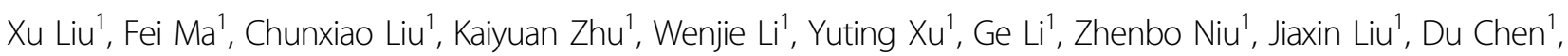 \\ Zhigao Li ${ }^{1}$, Yingqiang $\mathrm{Fu}^{1}$ and Cheng Qian ${ }^{1,2}$
}

\begin{abstract}
Ubiquitin-conjugating enzyme E2O (UBE2O) is a large E2 ubiquitin-conjugating enzyme that possesses both E2 and E3 ligase activities. Ectopic UBE2O overexpression is associated with a variety of human diseases, especially cancers. However, the expression profile and functional biology of UBE2O in human breast cancer (BC) remain unclear. In this study, we found that UBE2O was significantly overexpressed in human BC tissues and cells. Patients with high UBE2O expression tended to have a high risk of metastasis and poor prognosis. In vitro assays revealed that UBE2O promoted $\mathrm{BC}$ cell proliferation and epithelial-mesenchymal transformation (EMT) and endowed BC cells with cancer stemness properties (CSPs). UBE2O knockdown in MDA-MB-231 cells suppressed tumour growth and lung metastasis in MDAMB-231 xenograft mouse models. Mechanistically, UBE2O functioned as a ubiquitin enzyme of AMPKa2, promoting its ubiquitination and degradation and thus activating the mTORC1 signal pathway and contributing to BC oncogenesis and metastasis. Furthermore, as a downstream factor of the UBE2O/AMPKa2/mTORC1 axis, the oncoprotein MYC transcriptionally promoted UBE2O and formed a positive feedback loop in human BC. Collectively, our study demonstrated that UBE2O/AMPKa2/mTORC1-MYC forms a positive feedback loop in human BC cells that regulates BC cell proliferation and EMT and endows BC cells with CSPs.
\end{abstract}

\section{Introduction}

Ubiquitin-conjugating enzyme $\mathrm{E} 2 \mathrm{O}$ (UBE2O) is an E2 ubiquitin-conjugation enzyme that acts as a combination of E2 and E3 enzymes and has both E2 and E3 activities ${ }^{1}$. It is generally expressed in mammalian tissues but is present at higher levels in the brain, heart, skeletal muscle and liver ${ }^{2}$. The deregulation of UBE2O is associated with several human diseases. UBE2O mediates SMAD6 ubiquitination during bone morphogenetic

Correspondence: Cheng Qian (qiancheng@ems.hrbmu.edu.cn)

${ }^{1}$ Department of breast cancer surgery, Harbin Medical University Cancer Hospital, Harbin Medical University, Harbin, Heilongjiang Province, China ${ }^{2}$ North China Translational Medicine Research Center of Harbin Medical University, Harbin Medical University, Harbin, Heilongjiang Province, China Edited by M. Herold protein signalling ${ }^{3}$ and inhibits TRAF6 K63-polyubiquitination, in turn preventing NF- $\mathrm{kB}$ signalling activation ${ }^{4}$. It can also control cellular clock function by ubiquitinating the transcription factor BMAL1 ${ }^{5}$. Abnormal UBE2O expression occurs in many types of malignant tumours ${ }^{6-8}$. However, the precise role of UBE2O in human breast cancer $(\mathrm{BC})$ remains unclear.

AMP-activated protein kinase (AMPK) is an intracellular inducer of energy and metabolism. It is a heterotrimer that includes a catalytic $\alpha$ subunit, a regulatory $\beta$ subunit and a $\gamma$ subunit ${ }^{9}$. AMPK activation can be regulated by the ATP/AMP ratio or phosphorylation ${ }^{10}$, and its major function is to inhibit anabolism and induce catabolism $^{11}$. AMPK is also an important regulator that controls cellular osmotic pressure and the cellular entry of

\section{(c) The Author(s) 2020}

(c) Open Access This article is licensed under a Creative Commons Attribution 4.0 International License, which permits use, sharing, adaptation, distribution and reproduction c. in any medium or format, as long as you give appropriate credit to the original author(s) and the source, provide a link to the Creative Commons license, and indicate if changes were made. The images or other third party material in this article are included in the article's Creative Commons license, unless indicated otherwise in a credit line to the material. If material is not included in the article's Creative Commons license and your intended use is not permitted by statutory regulation or exceeds the permitted use, you will need to obtain permission directly from the copyright holder. To view a copy of this license, visit http://creativecommons.org/licenses/by/4.0/. 
many xenobiotics ${ }^{12-14}$. The ectopic expression of AMPK is often related to a series of human diseases, especially cancers. Previous studies demonstrated that AMPK $\alpha 2$ downregulation or activity reduction exist in human kidney, ovarian and $\mathrm{BCs}^{15,16}$. The antitumour functions of AMPK could be summarised as follows: (1) AMPK can regulate the Hippo pathway, which inhibits cell growth; ${ }^{17}$ (2) AMPK is a downstream target of LKB1, a well-known tumour suppressor; $^{18}$ (3) AMPK activation inhibits ACCA, blocks lipogenesis and reduces tumour growth; ${ }^{19}$ and (4) AMPK can phosphorylate the oncostatin TSC2 and the mTORC1 partner Raptor, thus inhibiting the mTOR pathway ${ }^{20,21}$. However, other studies reported that AMPK activation had a protumour function in RAS-transformed fibroblasts and astrocytes and promoted tumour cell survival ${ }^{22,23}$. AMPK can also alleviate cancer cell metabolic stress and apoptosis via mitochondrial pathways ${ }^{24,25}$. Therefore, the role of AMPK in BC needs to be researched further.

In this study, we demonstrated that UBE2O was significantly overexpressed in $\mathrm{BC}$ tissues and cells. Patients with high UBE2O expression tended to have a high risk of metastasis and poor prognosis. Functional assays proved that UBE2O promoted $\mathrm{BC}$ cell proliferation and epithelial-mesenchymal transformation (EMT) and conferred BC cells with cancer stemness properties (CSPs). Mechanistically, UBE2O acts as a ubiquitin enzyme of AMPK $\alpha 2$, facilitating its ubiquitination and degradation and thus activating the mTORC1 signalling pathway and contributing to $\mathrm{BC}$ oncogenesis and metastasis. Pharmacological intervention of UBE2O inhibited its protumour activities in $\mathrm{BC}$ cells by recovering $\mathrm{AMPK} \alpha 2$, indicating that $\mathrm{UBE} 2 \mathrm{O}$ could be a promising target for $\mathrm{BC}$ therapy. Furthermore, as a downstream target of the UBE2O/ AMPK $\alpha 2 / m$ TORC1 pathway, MYC transcriptionally promoted UBE2O expression, which indicated that this axis constitutes a positive feedback loop.

\section{Materials and methods}

\section{$B C$ cells and specimens}

MDA-MB-231, MCF-7 and T-47D cells were purchased from the cell bank of the Chinese Academy of Sciences (Shanghai, China). MDA-MB-453, MDA-MB-468, SKBR-3, Hs578T, BT-549 and MCF-10A cells were purchased from the BeNa Culture Collection (Beijing, China). MDA-MB-231, MDA-MB-453, MDA-MB-468, T-47D, MCF-7, SK-BR-3 and Hs578T cells were cultivated in DMEM (Gibco, USA) with 10\% fetal bovine serum (FBS). BT-549 cells were maintained in RPMI 1640 (Gibco, USA) with $10 \%$ FBS. MCF-10A cells were cultured with an MEGM kit containing cholera toxin. All cell lines were incubated at $37^{\circ} \mathrm{C}$ in a humidified atmosphere containing $5 \%$ carbon dioxide and $95 \%$ oxygen.

Fresh $\mathrm{BC}$ tissues and paired normal tissues were acquired from $\mathrm{BC}$ patients $(n=50)$ who underwent surgery at the Harbin Medical University Cancer Hospital between April and September 2016. All tissue specimens were collected after resection and stored at $-80^{\circ} \mathrm{C}$ immediately. One hundred formalin-fixed, paraffinembedded primary BC specimens were also obtained from the Pathology Department of the Harbin Medical University Cancer Hospital from 2012 to 2013. All of the patients above had complete clinicopathological information. Patients who underwent adjuvant chemotherapy, immunotherapy or radiotherapy before surgery and those with recurrent tumours, metastatic disease, bilateral tumours, or other previous tumours were excluded. Our study was approved by the Research Ethics Committee of Harbin Medical University. Informed consent was signed by all patients who participated in this study.

\section{Real-time quantitative PCR (qRT-PCR)}

TRIzol reagent (Catalogue Number 15596018, Invitrogen, China) was used to extract total RNA, and cDNA was synthesised with a Rever Tra Ace qPCR kit (Catalogue Number FSQ-201, TOYOBO, Japan). qRT-PCR was conducted using SYBR Green Real-Time PCR Master Mix (Catalogue Number QPK-201, TOYOBO, Japan) on a CFX96 Touch Detection System (Bio-Rad, USA). The $2^{-\Delta \Delta C t}$ method was used to quantify gene expression. GAPDH was used as a reference gene. The sequences of the primers were as follows: GAPDH, 5'-GGAGCGA GATCCCTCCAAAAT- $3^{\prime}$ (F) and $5^{\prime}$-GGCTGTTGTCA TACTTCTCATGG-3' (R); UBE2O, 5'-GAATCCAA AACCAAGAGCGAAG- $3^{\prime}$ (F) and 5'-TCATCTCTG CCTTCTTTTAGCA-3'(R); MYC, 5'-GGCTCCTGG CAAAAGGTCA-3'(F) and 5'-CTGCGTAGTTGTGCT GATGT-3'(R).

\section{Cell counting kit-8 (CCK-8) and colony formation assays}

For the CCK- 8 assay, cells $\left(2 \times 10^{3}\right.$ per well $)$ were seeded into each well of 96-well plates and incubated in culture medium. At each pre-set time, $10 \mu \mathrm{l}$ of CCK- 8 solution (Catalogue Number C0038, Beyotime, China) was added to each well containing $90 \mu \mathrm{l}$ of culture medium. Then, the plates were incubated at $37^{\circ} \mathrm{C}$ for $2 \mathrm{~h}$, and absorbance was detected at $570 \mathrm{~nm}$.

For the colony formation assay, the indicated BC cells were seeded into six-well plates (500 cells per well), and the medium was refreshed every 2 days. Two weeks later, the cells were fixed with formalin for $30 \mathrm{~min}$. Crystal violet (Catalogue Number C8470, Solarbio, China) was applied to stain the cells, and photographs were taken with the FluorChem M system (ProteinSimple, USA).

\section{Wound healing and invasion assays}

For the wound healing assay, cells were seeded into sixwell plates, and scratch wounds were made with sterile micropipette tips after the cells reached $100 \%$ confluence. 
At each pre-set time, the cells were washed with phosphate-buffered saline, and images were photographed under a microscope. Then, the migration rates of the indicated cells were measured and analysed.

For the invasion assay, the upper chambers of 24-well Transwell plates (Coring, USA) were coated with Matrigel (Catalogue Number 356234, Coring, USA). Cells $\left(1 \times 10^{5}\right)$ were suspended in $200 \mu \mathrm{l}$ of serum-free medium and seeded into the upper chambers of the Transwell plates. Then, $600 \mu \mathrm{l}$ of $10 \%$ FBS medium was added to the lower chambers, and the cells were incubated at $37^{\circ} \mathrm{C}$ for $24 \mathrm{~h}$. Afterwards, the chambers were fixed, and the cells were stained with crystal violet. Then, images were taken, and the cells were counted under a microscope.

\section{Sphere culture and sphere formation assays}

Cells were suspended in cancer stemness medium $(1 \times$ $10^{3}$ cells per $\mathrm{ml}$ ) consisting of DMEM/F-12 (Catalogue Number 12660012, Gibco, USA), $1 \times$ B27 (Catalogue Number 17504044, Invitrogen, USA), $20 \mathrm{ng} / \mathrm{ml}$ epidermal growth factor (Catalogue Number PHG0311, Invitrogen, USA), $20 \mathrm{ng} / \mathrm{ml}$ basic fibroblast growth factor (Catalogue Number PHG0263, Invitrogen, USA), and $2 \mathrm{~mm}$ L-glutamine (Catalogue Number 25030081, Invitrogen, USA). Then, the cells were seeded into ultra-low attachment plates (Thermo Scientific, USA). The culture medium was replaced every $48 \mathrm{~h}$. Two weeks later, the stem spheres were imaged and counted under a microscope.

\section{Immunohistochemistry (IHC) and immunofluorescent staining}

IHC assays were performed on paraffin-embedded specimens from $\mathrm{BC}$ patients and mice with a standard streptavidin-peroxidase complex method. The staining results were evaluated and scored with a standard histochemistry score (H-SCORE) independently by three pathologists.

Immunofluorescent staining assays were performed as previously described ${ }^{26}$. In brief, cells were fixed, permeabilized with $0.5 \%$ Triton-100 (Solarbio, China) and blocked with $5 \%$ bovine serum albumin. Afterwards, the cells were incubated with the following primary antibodies (Cell Signaling Technology, USA): anti-CDH1 (14472) and anti-vimentin (5741). Then, the cells were incubated with the corresponding secondary antibodies (CST, USA) and 4',6-diamidino-2-phenylindole (DAPI) (Thermo Fisher Scientific, USA). Finally, the staining results were imaged by confocal microscopy.

\section{Western blot and cycloheximide assays}

For the western blot assay, $\mathrm{BC}$ cells and tissues were lysed with radioimmunoprecipitation assay (RIPA) (Catalogue Number P0013B, Beyotime, China) buffer containing phenylmethylsulfonyl fluoride (PMSF) (Catalogue
Number ST506, Beyotime, China). The lysate was collected, and western blot assays were conducted as previously described ${ }^{27}$. The following antibodies were used for these experiments: UBE2O (15812-1-AP), AMPK $\alpha 1$ (10929-2-AP), AMPK 2 (18167-1-AP), CDH2 (22018-1AP), CD44 (15675-1-AP), ABCG2 (27286-1-AP), MYC (10828-1-AP) and $\beta$-actin (60008-1-Ig), which were purchased from the Proteintech Group. CDH1 (14472), vimentin (5741), Slug (9585), OCT4 (2750), Raptor (2280), PS792-Raptor (2083), S6K (2708) and PT389-S6K (9234) were purchased from CST.

For the cycloheximide (CHX) assay, MDA-MB-231 cells were seeded into six-well plates and cultured overnight. Then, CHX (200 $\mu$ g per ml) (Catalogue Number C76985G, Sigma, USA) was added to the culture medium. The cells were collected at scheduled times $(0,2,4,6$ and $8 \mathrm{~h})$ and subjected to western blotting.

\section{Lentiviral transfection}

To establish stable UBE2O overexpression cells, UBE2O expression lentiviral vectors and empty vectors (Genechem, China) were transfected into MCF-7 cells with polybrene $(8 \mu \mathrm{g}$ per $\mathrm{ml})$. Twenty-four hours later, the cells were selected using puromycin, and stable UBE2O expression cells were acquired.

To construct stable UBE2O or AMPK $\alpha 2$ knockdown cells, shRNAs targeting UBE2O and AMPK $\alpha 2$ were designed (Sigma, USA). The shRNA sequences were as follows: UBE2O-shRNA\#1: 5'-CCGGCGATGATTCCTA TGGCTTCTACTCGAGTAGAAGCCATAGGAATCAT CGTTTTT-3'; UBE2O-shRNA\#2: 5'-CCGGGACATCAA GAAGCTACAGGAACTCGAGTTCCTGTAGCTTCTT GATGTCTTTTT-3'; UBE2O-shRNA\#3: 5'-CCGGCGG GTCTCTTCTTCGATGATTCTCGAGAATCATCG AAGAAGAGACCCGTTTTT-3'; AMPK $\alpha 2$-shRNA\#1: 5'-CCGGCGCAGTTTAGATGTTGTTGGACTCGAGT CCAACAACATCTAAACTGCGTTTTT-3'; AMPK $\alpha 2-$ shRNA\#2: 5'-CCGGGTGGCTTATCATCTTATCATTC TCGAGAATGATAAGATGATAAGCCACTTTTT-3'; and AMPK $\alpha$ 2-shRNA\#3: 5'-CCGGCCCACTGAAACG AGCAACTATCTCGAGATAGTTGCTCGTTTCAG

TGGGTTTTT-3'. The efficiency of transfection was evaluated by qRT-PCR and western blotting.

\section{Plasmids and RNA interference}

Flag-tagged UBE2O, MYC-tagged AMPK $\alpha 2$ and Histagged ubiquitin plasmids were constructed by Genechem (Genechem, China). All constructs were verified by fulllength sequencing. siRNAs targeting MYC were purchased from GenePharma (Shanghai, China). Transient transfection was performed with Lipofectamine 2000 transfection reagent (Catalogue Number 11668019, Invitrogen, USA) according to the manufacturer's instructions. The sequences were as follows: MYC-RNAi\#1: 
5'-GAGGAUAUCUGGAAGAAAUTTAUUUCUUCCAGAUAUCCUCTT-3'; MYC-RNAi\#2: 5'-GCUUGUACC UGCAGGAUCUTTAGAUCCUGCAGGUACAAGCTT3'; and MYC-RNAi\#3: 5'-GGAAGAAAUCGAUGUUG UUTTAACAACAUCGAUUUCUUCCTT-3'.

\section{Immunoprecipitation (IP) and ubiquitination assays}

For IP assays, MDA-MB-231 cells were lysed with RIPA buffer containing PMSF, and the samples were separated by centrifugation. Afterwards, some of the lysate was taken for input, and the remainder was incubated with IgG or the appropriate antibodies at $4{ }^{\circ} \mathrm{C}$ overnight. Then, beads were added to the mixture, rotated for $4 \mathrm{~h}$ and washed with Tris-buffered saline (TBS) containing PMSF. Finally, the samples were boiled and subjected to western blotting. For co-immunoprecipitation (Co-IP) assays, MCF-7 cells were simultaneously transfected with Flagtagged UBE2O and MYC-tagged AMPK $\alpha 2$ plasmids (GeneChem, China). Forty-eight hours later, the cells were collected and lysed, and the cell lysates were subjected to IP as described above.

The in vitro ubiquitination assays were performed as described in a previous report ${ }^{28}$. In brief, His-tagged ubiquitin plasmids (GeneChem, China) were transfected into the indicated cells and cultured for $48 \mathrm{~h}$. Then, the cells were collected, and the cell lysates were subjected to IP as described above.

\section{Chromatin immunoprecipitation (ChIP)}

A ChIP assay kit (Catalogue Number, P2078, Beyotime, China) was used according to the manufacturer's instructions. In brief, MDA-MB-231 and MCF-7 cells were treated with formaldehyde at a final concentration of $1 \%$ to cross-link the target proteins and DNA. Then, a glycine solution was added to terminate the reaction. The samples were separated by centrifugation at $1000 \times g$ for $2 \mathrm{~min}$. Then, the cells were resuspended in sodium dodecyl sulphate lysis buffer with PMSF and lysed with an ultrasonic cell disruptor on ice. Afterwards, the DNA was extracted and cleaned using a DNA depuration kit (Catalogue Number D0033, Beyotime, China). Next, the samples were incubated with anti-MYC (CST, USA) or IgG antibodies at $4{ }^{\circ} \mathrm{C}$ overnight, and protein $\mathrm{A}$ was used to precipitate the compound. Finally, the DNA was purified, and qRT-PCR was performed to detect the promoter fragments of UBE2O. The primers for the UBE2O promoter were $5^{\prime}$-TCCCAGGTTCAAGCGATTTG-3' (F) and 5'-CATGGCGAAACCCCATCTCTACT-3' (R).

\section{Luciferase reporter assay}

A double luciferase assay system (Promega, USA) was used according to the manufacturer's protocol. In brief, wild-type or mutant-type UBE2O promoter luciferase reporter plasmids were transfected into $293 \mathrm{~T}$ cells, and different amounts of MYC plasmids were transfected into $293 \mathrm{~T}$ cells as well. Forty-eight hours later, the cells were lysed with passive lysis buffer, and luciferase assays were performed. Firefly luciferase activity normalised to Renilla luciferase activity was used as an internal control.

\section{Animal study}

All animal studies were approved by the Medical Experimental Animal Care Commission of Harbin Medical University. For the tumourigenesis assay, six-weekold female BALB/c nude mice (Beijing Vital River Laboratory Animal Technology Co., China) were randomised into two groups $(n=6)$. MDA-MB-231-Luc cells $\left(5 \times 10^{5}\right)$ transfected with sh-UBE2O\#1 and sh-NC were injected into the mammary fat pads of mice, respectively. Tumour growth was measured every 5 days. Seven weeks after injection, the mice were imaged using an Xenogen IVIS Spectrum Imaging System (Caliper Life Sciences, USA), and the tumours were collected for IHC. The tumour volumes were evaluated with the following formula: $1 / 2$ (length $\times$ width $^{2}$ ). For the tumour metastasis model, six-week-old female BALB/c nude mice $(n=6)$ were randomised into two groups. MDA-MB-231-Luc cells $\left(5 \times 10^{5}\right)$ transfected with sh-UBE2O\#1 and sh-NC were injected into the tail vein of the mice, respectively. Seven weeks after injection, the lungs were imaged and collected for haematoxylin and eosin staining. The sample size of each group was determined according to the previous studies ${ }^{29-31}$.

\section{Statistical analysis}

All the data are presented as the means \pm SDs from at least three independent experiments. Statistical analysis was performed using Student's $t$ test or one-way analysis of variance and the variances between the groups which were being statistically compared were similar. For animal studies, no blinding was used. The chi-square test was used to analyse the relationship between UBE2O expression and the clinicopathological features of $\mathrm{BC}$ patients. The Kaplan-Meier method and log-rank test were employed to draw survival curves. $P<0.05$ indicated statistical significance, which was evaluated using SPSS 21.0 software (Statistical Product and Service Solutions, USA).

\section{Results}

UBE2O was commonly overexpressed in BC tissues and associated with the prognosis of $\mathrm{BC}$ patients

We sought to detect UBE2O expression in 50 pairs of $\mathrm{BC}$ tissues and corresponding normal tissues by qRT-PCR and western blotting. The results showed that UBE2O was significantly upregulated in cancer tissues compared with corresponding normal tissues (Fig. 1a, b). However, no differences in UBE2O expression were found between the 
A

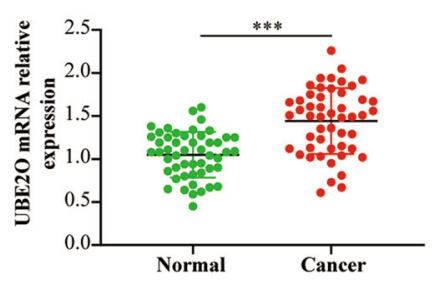

D
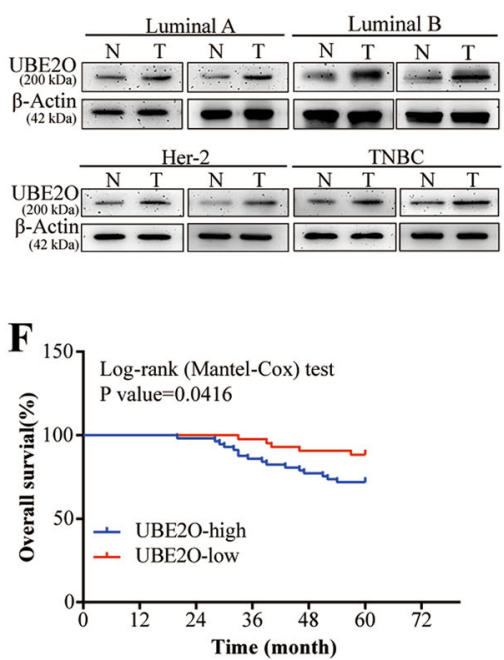

H

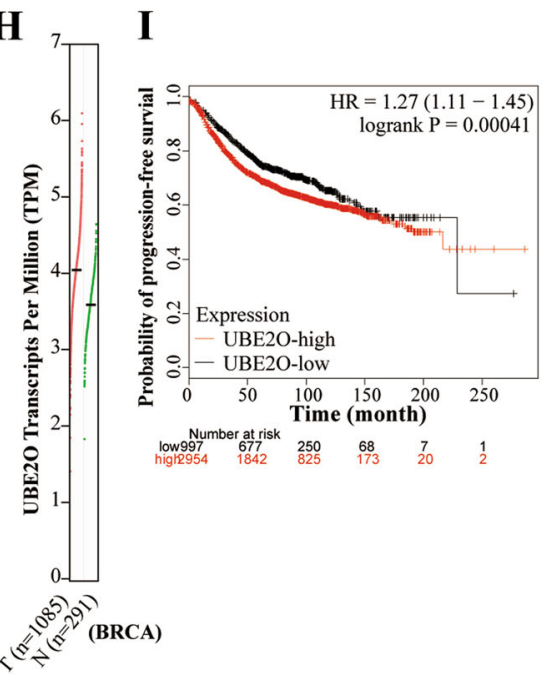

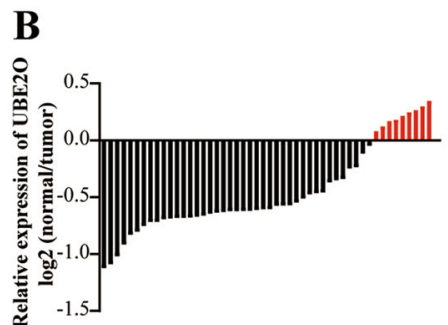

C

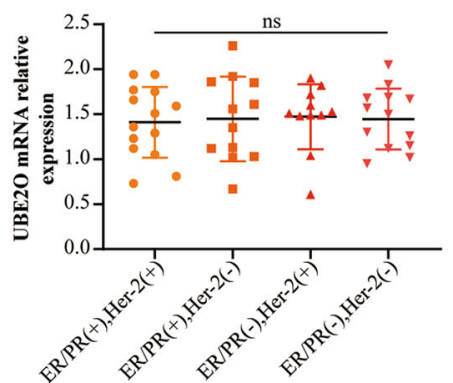

E

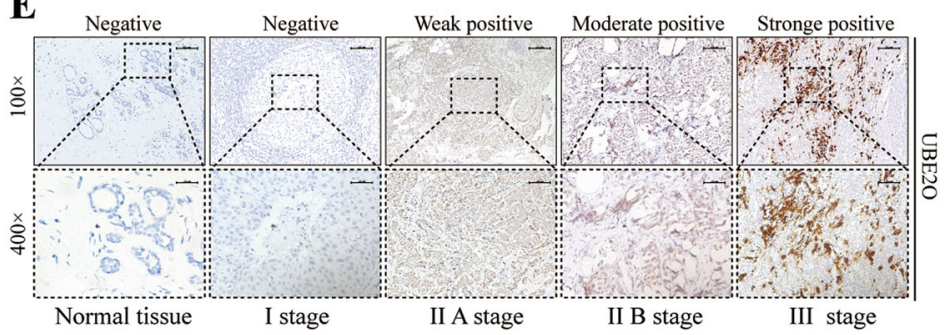

G

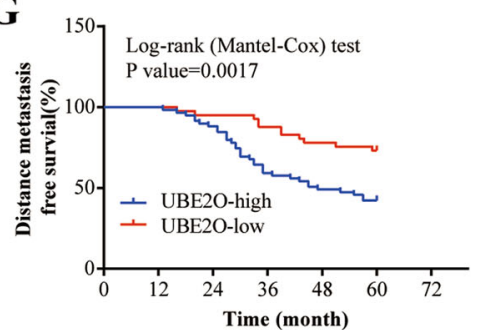

J

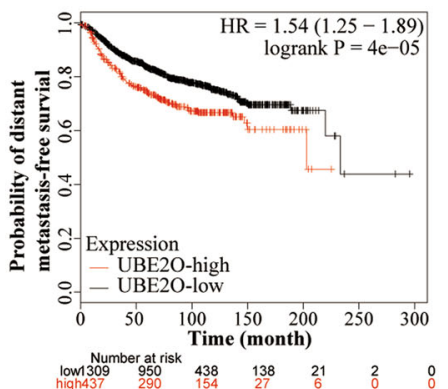

K

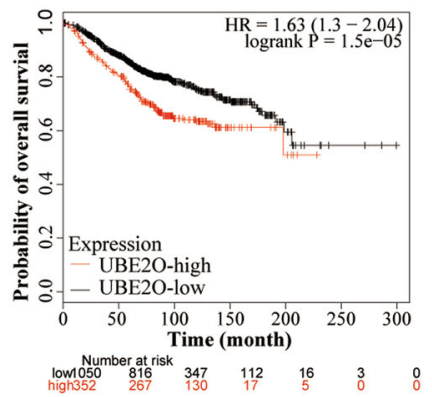

Fig. 1 UBE2O was commonly overexpressed in BC tissues and associated with the prognosis of $B C$ patients. $\mathbf{a}$, $\mathbf{b}$ qRT-PCR analysis of UBE2O expression in BC tissues and adjacent normal tissue from BC patients $(n=50)$. c qRT-PCR was applied to analyse discrepancies in UBE2O expression in different subtypes of BC tissues $(n=50)$. $\mathbf{d}$ The protein expression level of UBE2O was analysed in eight cases of different subtypes of $B C$ tissues and corresponding normal tissues by western blotting; $\beta$-actin served as a reference. e UBE2O expression in different clinical stages of BC tissues and normal mammary tissue was determined by immunohistochemistry $(\mathrm{HC})$ staining. Representative images were shown (upper: magnification $\times 100$, Scale bar, 100 um; lower: magnification $\times$ 400, Scale bar, $20 \mu \mathrm{m})$. f, g Kaplan-Meier survival curve analysis revealed that patients with high UBE2O expression had poorer overall survival (OS) and distance metastasis-free survival (DMFS) than those with low UBE2O expression. $\mathbf{h}$ The GEPIA database was used to analyse discrepancies in transcripts from BC patient tissues $(n=1085)$ and normal mammary tissues $(n=291)$. $\mathbf{i}-\mathbf{k}$ Progression-free survival (PFS), DMFS and OS of BC patients with high or low UBE2O expression were analysed by the KMplot database. ${ }^{*} p<0.05,{ }^{* *} p<0.01,{ }^{* * *} p<$ 0.001. The data represent at least three independent experiments. 
four subtypes of BC tissues (Fig. 1c, d). IHC staining was then used to explore the clinical significance of UBE2O expression in BC patients. Representative images of UBE2O expression in different clinical stages of BC tissues and normal breast tissues are shown in Fig. 1e. The clinicopathological features of these patients are shown in Table 1 . We found that the UBE2O state was positively correlated with tumour size $(p=0.014)$, axillary lymph node status $(p=0.038)$ and clinical stage $(p=0.019)$. Furthermore, the log-rank test survival analysis confirmed that patients with high UBE2O expression had worse overall survival $(\mathrm{OS})(p=0.0416)$ and distant metastasisfree survival (DMFS) $(p=0.0017)$ (Fig. 1f, g). Given the limited number of patients enrolled in our study, we applied two standard databases to examine the UBE2O status in BC patients. Data from Gene Expression Profiling Interactive Analysis showed that UBE2O transcription was upregulated in tumour tissues (Fig. 1h). Survival

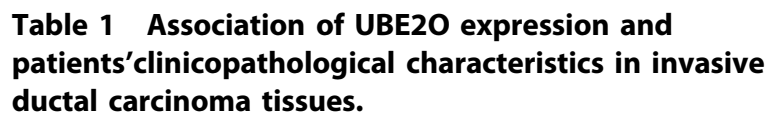

\begin{tabular}{|c|c|c|c|c|}
\hline \multirow[t]{2}{*}{ Characteristics } & \multicolumn{2}{|c|}{ UBE2O expression } & \multirow[t]{2}{*}{ No. } & \multirow[t]{2}{*}{$P$ value } \\
\hline & High & Low & & \\
\hline Age & & & 100 & 0.589 \\
\hline$\leq 40$ & $4(50.00 \%)$ & $4(50.00 \%)$ & & \\
\hline$>40$ & $55(59.78 \%)$ & $37(40.22 \%)$ & & \\
\hline$E R / P R$ & & & 100 & \\
\hline$(+)$ & $28(50.91 \%)$ & 27 (49.09\%) & & 0.069 \\
\hline$(-)$ & $31(68.89 \%)$ & $14(31.11 \%)$ & & \\
\hline HER-2 & & & 100 & 0.290 \\
\hline $0-2(+)$ & $17(68.00 \%)$ & $8(32.00 \%)$ & & \\
\hline $3(+)$ & $42(56.00 \%)$ & $33(44.00 \%)$ & & \\
\hline Tumour size & & & 100 & 0.014 \\
\hline $\mathrm{D} \leq 2 \mathrm{~cm}$ & $20(45.45 \%)$ & $24(54.55 \%)$ & & \\
\hline $\mathrm{D}>2 \mathrm{~cm}$ & $39(69.64 \%)$ & $17(30.36 \%)$ & & \\
\hline Axillary lymph node metastasis & & & 100 & 0.038 \\
\hline No & $31(50.82 \%)$ & $30(49.18 \%)$ & & \\
\hline Yes & $28(71.79 \%)$ & $11(28.21 \%)$ & & \\
\hline Clinical stages & & & 100 & 0.019 \\
\hline $\mid-\| A$ & $34(50.75 \%)$ & $33(49.25 \%)$ & & \\
\hline$\|\mathrm{B}-\|||$ & $25(75.76 \%)$ & $8(24.24 \%)$ & & \\
\hline Histological grade & & & 100 & 0.089 \\
\hline 1-2 grade & $40(54.05 \%)$ & $34(45.95 \%)$ & & \\
\hline 3 grade & 19 (73.08\%) & 7 (26.92\%) & & \\
\hline
\end{tabular}

Statistically significant difference $(P<0.05)$ are indicated in bold information from KMplot indicated that $\mathrm{BC}$ patients with high UBE2O expression had worse progression-free survival (PFS), DMFS and OS (Fig. 1i-k). Collectively, these results indicated that $\mathrm{UBE} 2 \mathrm{O}$ was highly expressed in $\mathrm{BC}$ tissues and negatively correlated with the prognosis of $\mathrm{BC}$ patients.

\section{Upregulation of UBE2O promoted BC cell proliferation and EMT}

To explore the function of UBE2O in $\mathrm{BC}$ cells, western blot assays were performed with different $\mathrm{BC}$ cell lines to examine UBE2O expression. As shown in Fig. 2a, UBE2O was obviously overexpressed in the highly metastatic cell line MDA-MB-231, and the expression was relatively lower in the low metastatic cell line MCF-7. However, the mammary epithelial cell line MCF-10A had the lowest UBE2O expression compared with the other $\mathrm{BC}$ cell lines. To directly test the putative cancerogenic function of UBE2O, MDA-MB-231 cells were infected with three specific short hairpin RNAs (shRNAs) with a lentivirusmediated system to establish UBE2O knockdown cell lines. Meanwhile, MCF-7 was used to establish high UBE2O expression cell lines $\left(\mathrm{MCF}^{\mathrm{OE}-U B E 2 O}\right)$. The transfection efficiency of the two cell lines was tested by western blotting and qRT-PCR. As shown in Fig. S1a$\mathrm{S} 1 \mathrm{~b}$, sh-UBE2O\#1 and sh-UBE2O\#3 yielded a relatively better UBE2O knockdown efficiency in MDA-MB-231

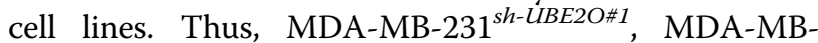
$231^{\text {sh-UBE2O\#3 }}$ and MCF-7 $7^{O E-U B E 2 O}$ cells were established and applied for subsequent investigation. Next, we performed CCK- 8 assays to detect the effect of UBE2O on $\mathrm{BC}$ cell proliferation. The results revealed that UBE2O knockdown reduced the proliferation ability of MDAMB-231 cells. Conversely, UBE2O overexpression significantly promoted MCF-7 cell growth in vitro (Fig. 2b, Fig. S1c). Colony formation assays also exhibited similar results (Fig. 2c, Fig. S1d). To further explore the correlation between $\mathrm{UBE} 2 \mathrm{O}$ status and tumour proliferation in human BC, Ki-67 expression in BC patients was detected by IHC and analysed by chi-square test. The results showed that the UBE2O status was positively associated with $\mathrm{Ki}-67$ expression (Ki-67 $>20 \%$ was regarded as a high expression level) in these BC patients (Fig. 2d). Finally, MDA-MB-231 $1^{\text {sh-NC }}$ and MDA-MB-231 ${ }^{\text {sh-UBE2O\#1 cells }}$ were injected into the mammary fat pads of $B A L B / c$ nude mice. As expected, UBE2O knockdown markedly suppressed xenograft tumour size (Fig. 2e, f) and yielded better tumour-free survival (Fig. $2 \mathrm{~g}$ ). In conclusion, these results confirmed that UBE2O promoted $\mathrm{BC}$ cell proliferation both in vitro and in vivo.

Then, we explored the effect of UBE2O on EMT, which is a crucial process in tumour metastasis. In wound healing assays, UBE2O knockdown decreased the migration of MDA-MB-231 cells, and UBE2O overexpression 


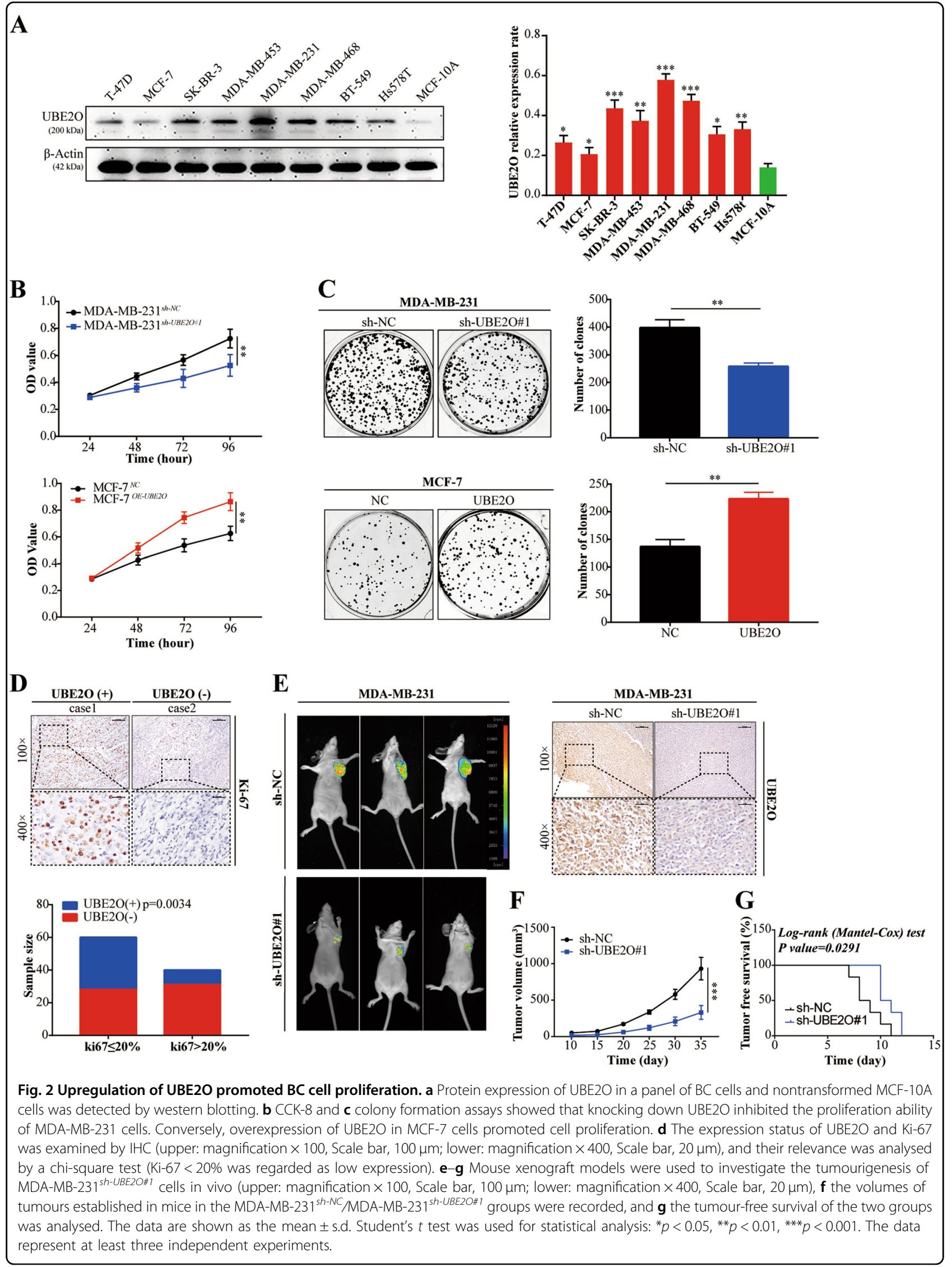


increased the migration capability of MCF-7 cells (Fig. 3a, Fig. S1e). In Matrigel-coated Transwell assays, UBE2O knockdown suppressed the invasive ability of MDA-MB231 cells, and UBE2O overexpression promoted the invasion of MCF-7 cells (Fig. 3b, Fig. S1f). Furthermore, western blot and immunofluorescence (IF) assays revealed that the epithelial marker $\mathrm{CDH} 1$ was upregulated, but the expression of $\mathrm{CDH} 2$, vimentin and slug was reduced in MDA-MB-231 ${ }^{\text {sh-UBE2O\#1 }}$ cells. The opposite results occurred in MCF-7 ${ }^{\mathrm{OE}-U B E 2 O}$ cells (Fig. 3c, d). To investigate the prometastasis effect of UBE2O in vivo, lung metastasis mouse models were established through tail vein injection in another group of nude mice. The results revealed that the mice injected with MDA-MB-231 ${ }^{\text {sh- }}$ ${ }^{U B E 2 O \# 1}$ cells exhibited fewer lung metastasis nodes than the mice injected with MDA-MB-231 ${ }^{\text {sh-NC }}$ cells (Fig. 3e). In conclusion, these results demonstrated that UBE2O promoted BC cell EMT and metastasis both in vitro and in vivo.

\section{UBE2O could induce BC cell CSPs}

Given that CSPs play critical roles in $\mathrm{BC}$ relapse and metastasis, further studies were carried out to determine whether UBE2O could mediate CSPs in BC cells. Cell stemness sphere assays showed that the sphere formation ability was obviously reduced in MDA-MB-231 ${ }^{\text {sh- }}$ $U B E 2 O \# 1$ cells, whereas MCF-7 ${ }^{\mathrm{OE}-U B E 2 O}$ cells exhibited greater CSP formation capability (Fig. 4a). Western blot analysis revealed that the CS markers CD44, ABCG2, OCT4 and MYC were significantly reduced in MDA-MB$231^{\text {sh-UBE2O\#1 }}$ cells compared with those in the NC group, whereas these markers were increased in MCF- $7^{O E-}$ ${ }^{U B E 2 O}$ cells (Fig. 4b). Finally, CD44 and MYC were detected by IHC staining to analyse their correlation with UBE2O expression in clinical samples. Significant positive relationships were found between UBE2O and CD44 or MYC (Fig. 4c, d). Collectively, these results confirmed that UBE2O endowed BC cells with CSPs in vitro.

\section{UBE2O-mediated AMPKa2 ubiquitination and degradation}

AMPK is a heterotrimer that includes an $\alpha$ subunit (AMPK $\alpha)$, a $\beta$ subunit $(\mathrm{AMPK} \beta)$ and a $\gamma$ subunit (AMPK $\gamma$ ). AMPK $\alpha$ also consists of two structural elements: AMPK $\alpha 1$ and AMPK $\alpha 2$. A previous study demonstrated that UBE2O is an AMPK $\alpha 2$-associated protein that ubiquitinates and degrades AMPK $\alpha 2$ in a series of transgenic mouse models of spontaneous can$\mathrm{cer}^{28}$. However, whether UBE2O could ubiquitinate and degrade AMPK $\alpha 2$ in human $B C$ has never been explored. We previously demonstrated that MDA-MB-231 cells had the highest level of UBE2O, so they were used for testing the effect of UBE2O on the stability of AMPK $\alpha$. CHX assays showed that AMPK $\alpha 2$ protein stability was remarkably enhanced, while AMPK $\alpha 1$ stability was not markedly altered (Fig. 5a). These results confirmed that AMPK $\alpha 2$, but not AMPK $\alpha 1$, was a downstream target of UBE2O and might play a part in UBE2O-dependent tumourigenesis. Next, endogenous IP assays were carried out on MDA-MB-231 cells to further explore the interaction between UBE2O and AMPK $\alpha 2$. Figure $5 \mathrm{~b}$ showed that UBE2O could combine with AMPK 22 in MDA-MB231 cells. Co-IP assays also showed that UBE2O could interact with AMPK $\alpha 2$ in MCF-7 cells (Fig. 5c). Then, His-tagged ubiquitin plasmids were transfected into the indicated cell lines, and the intracellular ubiquitination capacity was measured. As shown in Fig. 5d, the ubiquitination of AMPK $\alpha 2$ was significantly reduced in MDAMB-231 ${ }^{\text {sh-UBE2O\#1 }}$ cells and vice versa in MCF-7 cells. Finally, we used IHC staining to detect AMPK $\alpha 2$ in BC tissues, and a negative correlation between UBE2O expression and AMPK $\alpha 2$ was observed (Fig. 5e). Taken together, these results confirmed that UBE2O could mediate AMPK $\alpha 2$ ubiquitination and degradation in $\mathrm{BC}$ cells.

\section{UBE2O promoted proliferation, EMT and CSPs in BC cells through the UBE2O/AMPKa2/mTORC1 axis}

Next, AMPK substrates were detected in the two indicated BC cells, and Fig. 6a showed the enhanced expression of $\mathrm{p}$-Raptor and the decreased expression of $\mathrm{p}$-S6K in MDA-MB-231 ${ }^{\text {sh-UBE2O\#I }}$ cells, which indicated mTORC1 signalling inactivation. The opposite effects could be observed in MCF-7 $7^{\mathrm{OE}-U B E 2 O}$ cells. Previous studies have used many approaches to show that AMPK has tumoursuppressing functions. Among these, mTORC1 may be the most critical downstream target. AMPK could directly phosphorylate Raptor, the mTORC1-binding partner, and exert its antitumour effect by inactivating $\mathrm{mTORC}^{20,21}$. Our results were in accordance with these studies. However, the expression of another critical upstream regulator of the mTORC1 pathway, p-AKT, failed to show any alterations, which indicated that AMPK, but not AKT, mediated the function of UBE2O in BC. Next, AMPK $\alpha 2$ was stably knocked down in MDA-MB-231 cells (Fig. S2a$\mathrm{S} 2 \mathrm{~b})$, and the phenotypes of the $\mathrm{BC}$ cells were analysed. The results revealed that AMPK $\alpha 2$ knockdown could significantly reduce migration (Fig. 6b, Fig. S2c), invasion (Fig. 6c, Fig. S2d), proliferation (Fig. 6d, e, Fig. S2e-S2f) and CSPs (Fig. 6f) in MDA-MB-231 cells. Moreover, western blot assays confirmed that AMPK $\alpha 2$ knockdown also abolished the upregulation of an epithelial marker (CDH1) and the downregulation of mesenchymal markers (CDH2, vimentin) and CS markers (CD44, ABCG2, OCT4 and MYC) in MDA-MB-231 ${ }^{\text {sh-UBE2O\#1 }}$ cells (Fig. 6g). To further explore whether mTORC1 pathway activation was critical for the oncogenic activity of UBE2O in BC cells, MCF- $7^{N C} / \mathrm{MCF}-7^{\mathrm{OE}-U B E 2 O}$ cells were treated with rapamycin, an endogenous mTOR inhibitor. Western blot 


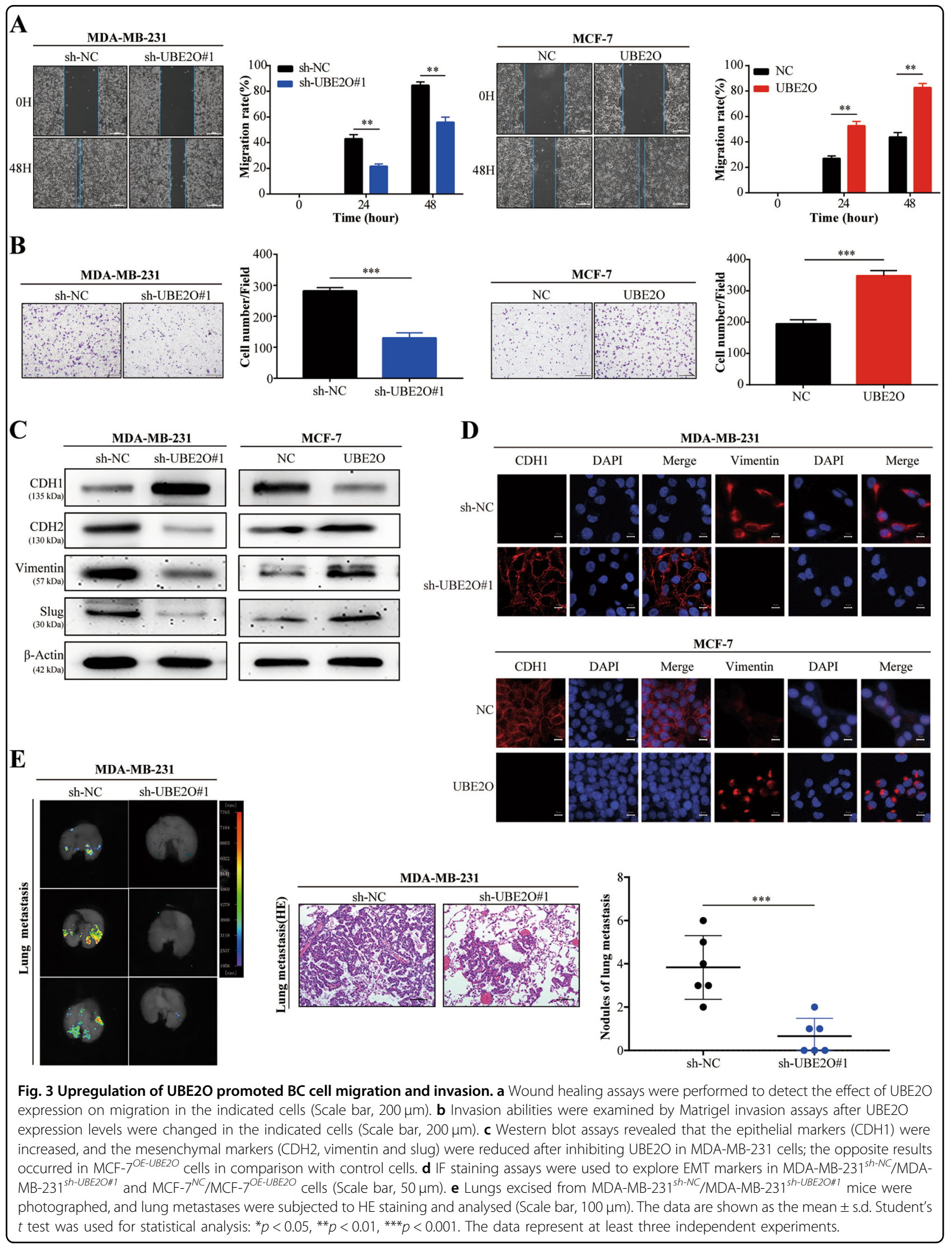




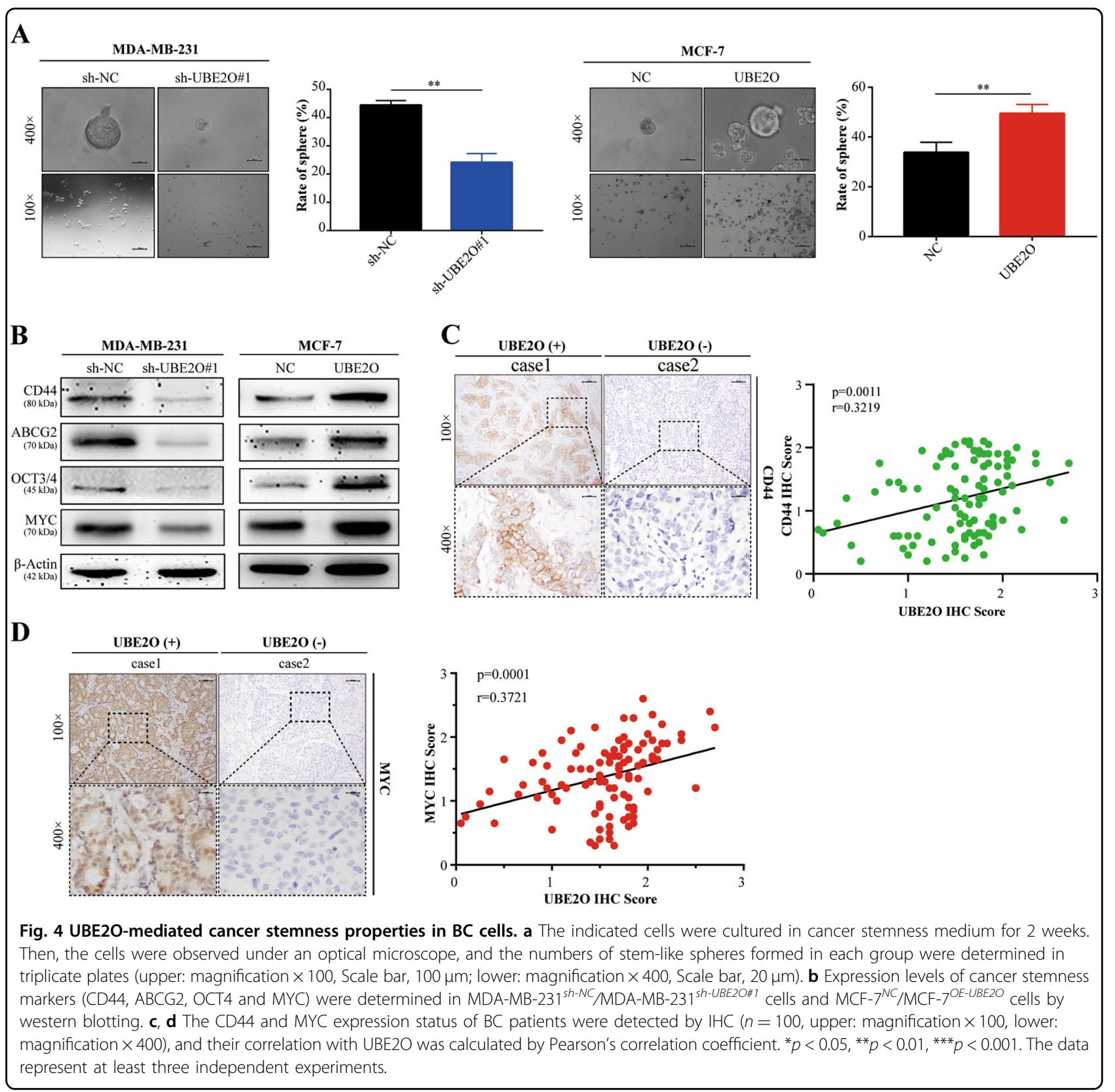

assays showed that the decreased $\mathrm{CDH} 1$ expression and enhanced mesenchymal ( $\mathrm{CDH} 2$ and vimentin) and cancer stemness (CD44, ABCG2, OCT4 and MYC) marker expression were abolished after treating MCF- $7^{O E-U B E 2 O}$ cells with rapamycin (Fig. 7a). Cellular functional experiments verified that the enhanced migration, invasion, proliferation and CSPs of MCF-7 $7^{\mathrm{OE}-U B E 2 O}$ cells were eliminated after treatment with rapamycin (Fig. 7b-f).

Given the crucial roles of UBE2O in BC, chemotherapy strategies targeting UBE2O might be a promising anticancer therapy. A previous investigation demonstrated that AMPK ubiquitination by UBE2O occurred via an intramolecular thioester mechanism. Arsenites can cross-link adjacent cysteines and, in turn, inhibit their ubiquitination $^{1}$. For this reason, a clinically relevant concentration of arsenic trioxide (ATO) (0.5-1 mM), which has been used for treating acute promyelocytic leukaemia, was adopted to inhibit UBE2O ubiquitination. The results in Fig. S3a showed that AMPK $\alpha 2$ expression was remarkably enhanced in MCF-7 cells treated with ATO at clinically relevant concentrations. This result showed that ATO could diminish the polyubiquitination degradation activity of UBE2O toward AMPK $\alpha 2$. Then, we treated MCF- $7^{N C}$ / MCF-7 $7^{\mathrm{OE}-U B E 2 O}$ cells with ATO and performed cellular functional experiments. Western blot assays confirmed that the upregulation of EMT and cancer stemness 


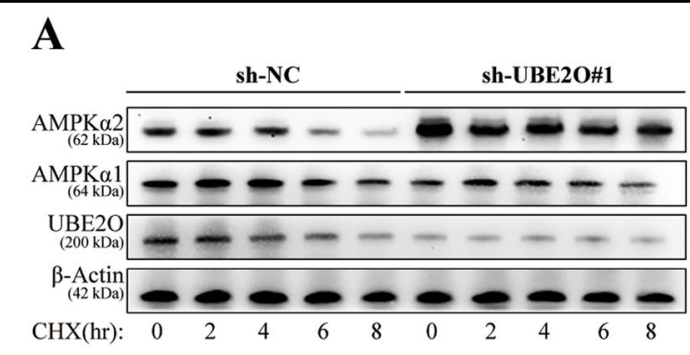

B

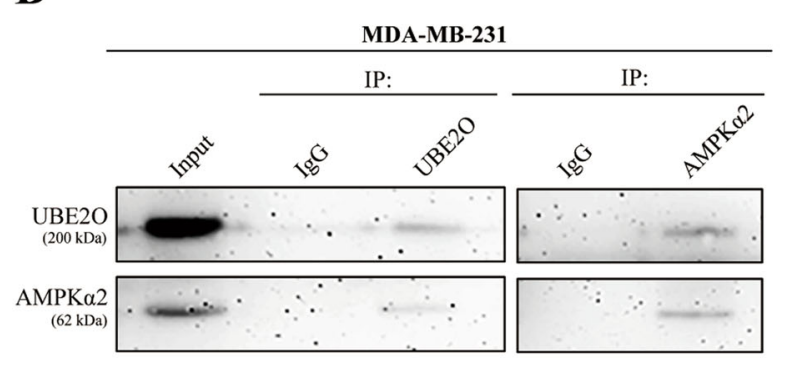

D
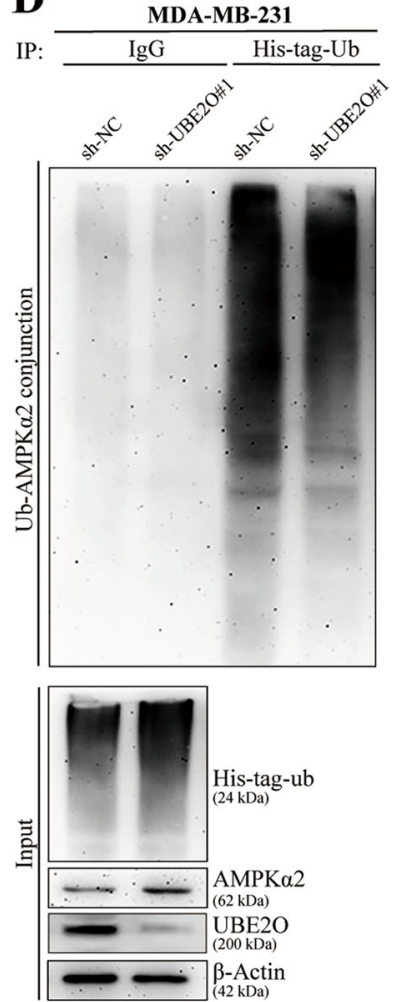
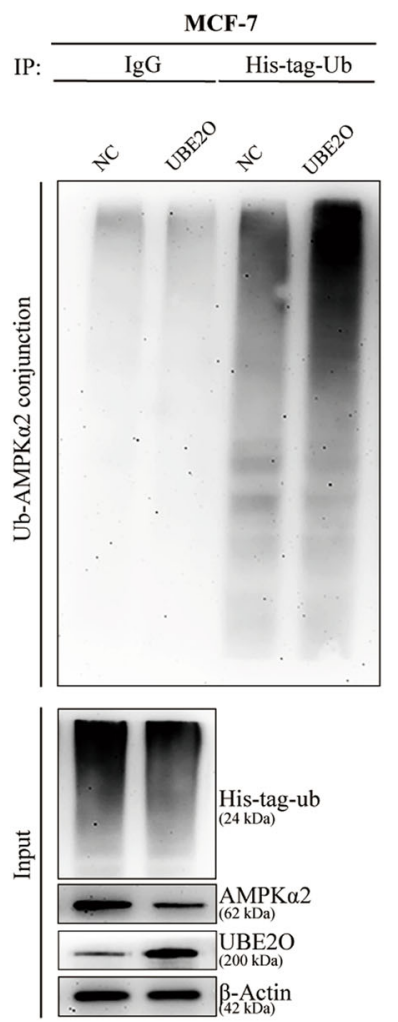
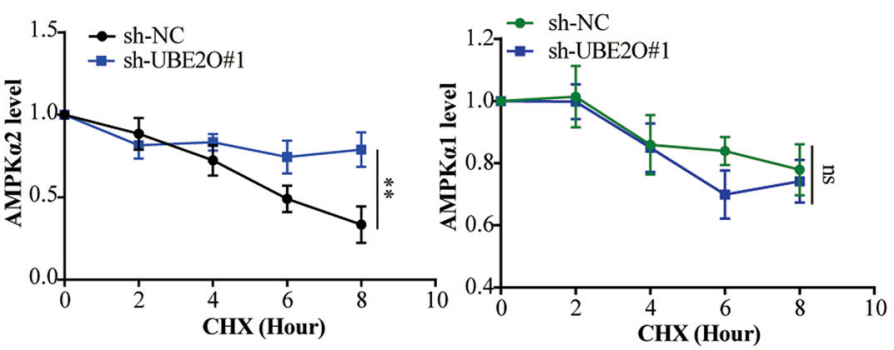

C

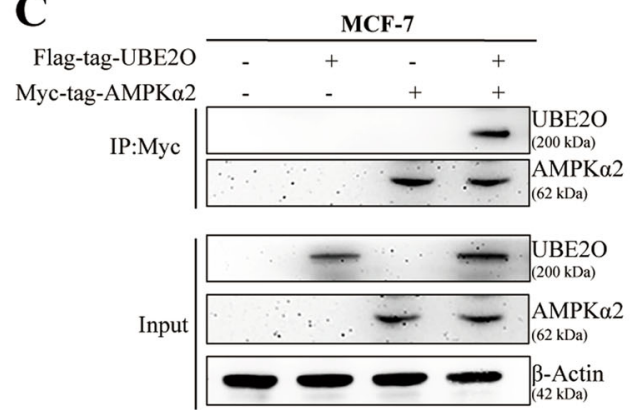

$\mathbf{E}$
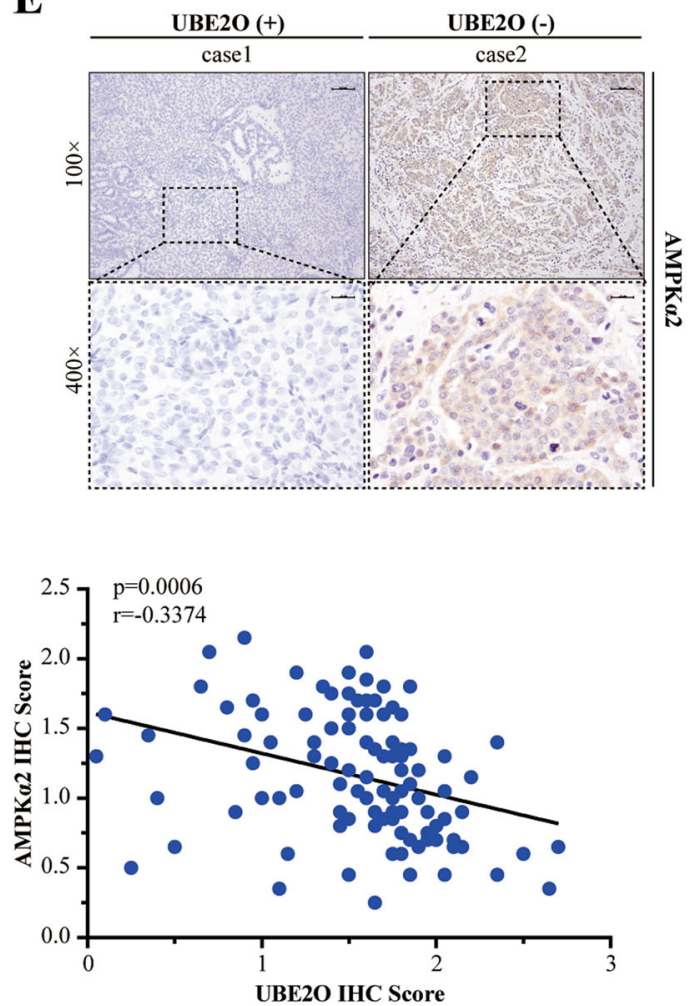

Fig. 5 UBE2O-targeted AMPKa2 ubiquitination and degradation. a Lysates from MDA-MB-231 $1^{\text {sh-NC}} / M D A-M B-231^{\text {sh-UBE2O\#1 }}$ cells treated with cycloheximide $(\mathrm{CHX})$ for the indicated times were subjected to western blotting. AMPKa2 and AMPKa1 expression levels were quantified and normalised to the intensity of the internal reference. $\mathbf{b}$ Lysates from MDA-MB-231 cells were immunoprecipitated with IgG, anti-UBE2O or antiAMPKa2 antibodies, and western blot assays were performed. c Flag-tagged UBE2O plasmids and MYC-tagged AMPKa2 plasmids were individually transfected or cotransfected into MCF-7 cells. Forty-eight hours after transfection, the cells were lysed, and the lysates were immunoprecipitated with MYC-tag antibodies. Then, western blot assays were performed using anti-UBE2O or anti-AMPKa2 antibodies. $\mathbf{d}$ Lysates from MDA-MB-231 ${ }^{\text {sh-NC}} / \mathrm{MDA}^{-}$ MB-231 $1^{\text {sh-UBE2O\#1 }}$ cells and MCF-7 ${ }^{\text {NC }}$ MCF-7 ${ }^{\text {UBE2O }}$ cells with His-tag ubiquitin were treated with MG132 $(10 \mu \mathrm{m})$ for $4 \mathrm{~h}$ and subjected to IP with IgG or His-tag antibodies and evaluated for ubiquitination with anti-AMPKa2 antibodies. e AMPKa2 expression in BC patients was detected by IHC, and its correlation with UBE2O was analysed by Pearson's correlation coefficient (upper: magnification $\times 100$, Scale bar, $100 \mu$ m; lower: magnification $\times 400$, Scale bar, $20 \mu \mathrm{m}) .{ }^{*} p<0.05,{ }^{* *} p<0.01,{ }^{* * *} p<0.001$. The data represent at least three independent experiments. 
A

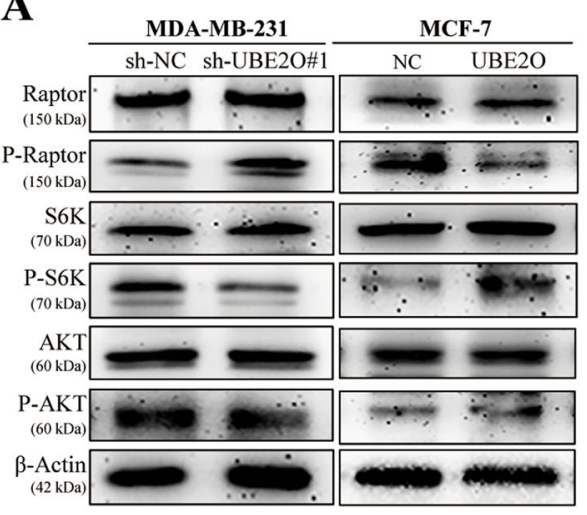

D
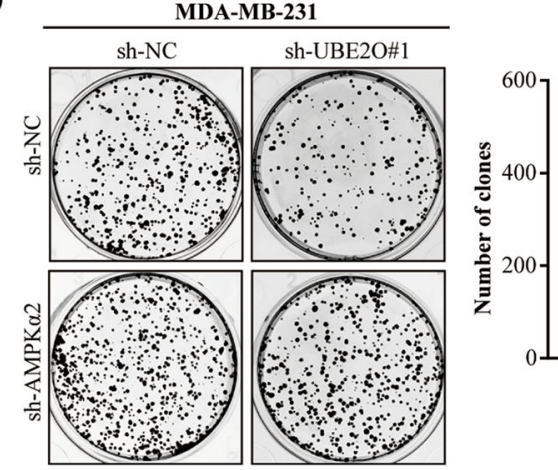

F

MDA-MB-231
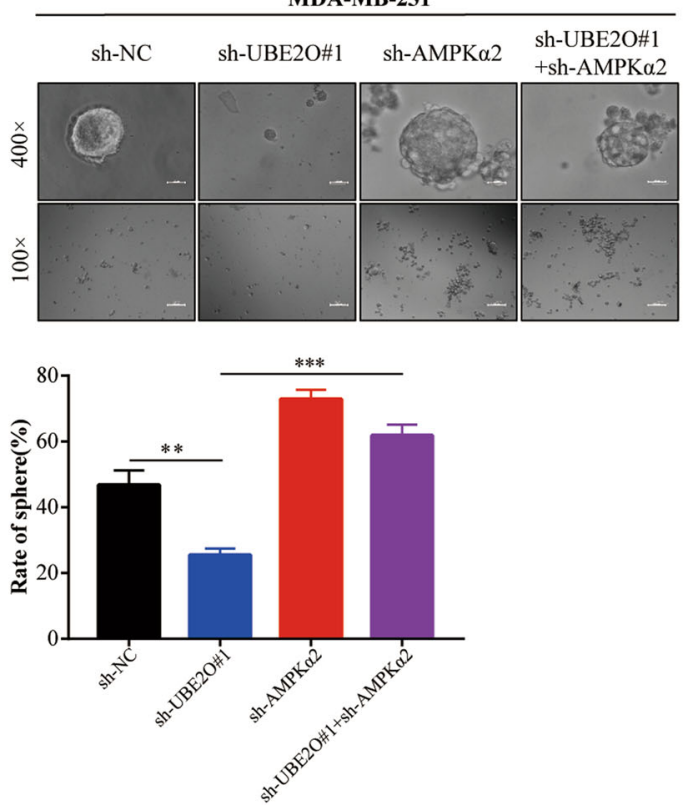

B

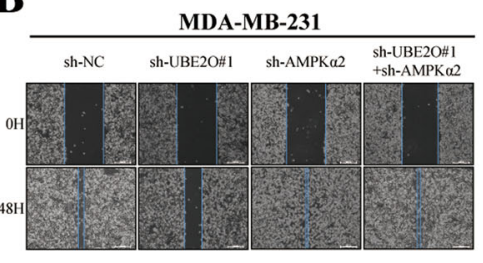

C

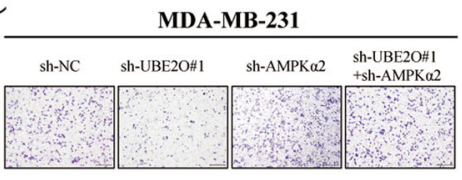

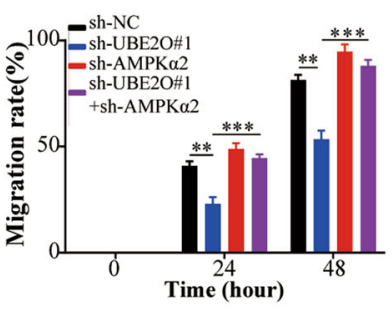

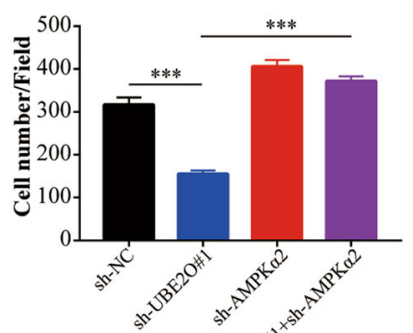

E

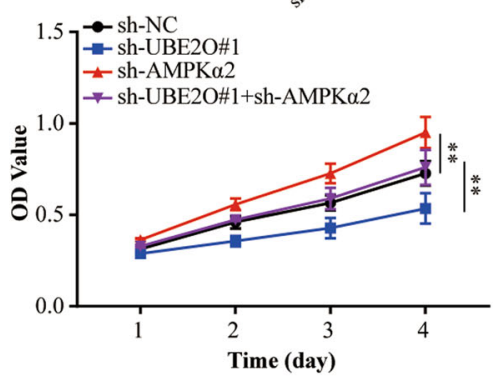

G

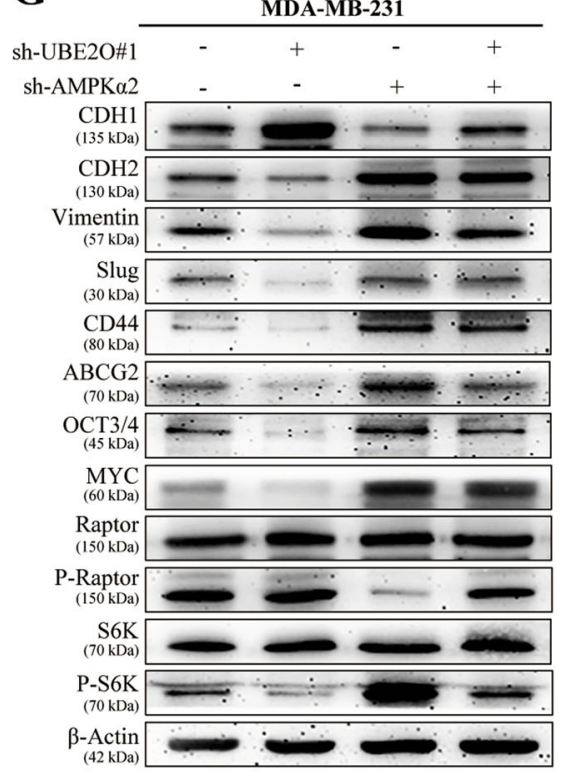

Fig. 6 (See legend on next page.) 
(see figure on previous page)

Fig. 6 UBE2O induced proliferation, EMT and CSPs in a manner dependent on the regulation of the UBE2O/AMPKa2/mTORC1 axis in BC. a Western blotting revealed that $\mathrm{p}$-Raptor expression was increased in MDA-MB-231 $1^{\text {sh-UBE2O\#1 }}$ cells, which indicated mTORC1 pathway inhibition. As a downstream effector of mTORC1, p-S6K expression was also decreased. The opposite results occurred in MCF-7 ${ }^{\text {EE-UBE2O }}$ cells. b-f AMPKa2 was knocked down in MDA-MB-231 $1^{\text {sh-NC}} /$ MDA-MB- $231^{\text {sh-UBE2O\#1 }}$ cells. Then, $\mathbf{b}$ scratch assays, $\mathbf{c}$ invasion assays, $\mathbf{d}$ colony formation assays, e CCK-8 assays and $\mathbf{f}$ sphere formation assays were performed (upper: magnification $\times 100$, Scale bar, $100 \mu \mathrm{m}$; lower: magnification $\times 400$, Scale bar, $20 \mu \mathrm{m}$ ). g Western blot assays were performed to detect the expression of EMT markers (CDH1, CDH2, vimentin and slug), CS markers (CD44, ABCG2, OCT3/4 and MYC) and mTORC1 pathway-related markers ( $\mathrm{p}$-Raptor and p-S6K) after AMPKa2 suppression in MDA-MB-231 ${ }^{\text {sh-NC }} / \mathrm{MDA}^{-M B}-231^{\text {sh-UBE2O\#1 }} \mathrm{cellls}$. The data are shown as the mean \pm s.d. Student's $t$ test was used for statistical analysis: ${ }^{*} p<0.05,{ }^{* *} p<0.01,{ }^{* * *} p<0.001$. The data represent at least three independent experiments.

markers in MCF-7 $7^{\mathrm{OE}-U B E 2 O}$ cells was blocked by ATO treatment (Fig. S3b). The functional experiments further showed that the enhanced migration, invasion, proliferation abilities and CSPs of MCF-7 $7^{\mathrm{OE}-U B E 2 O}$ cells were abolished by ATO treatment (Fig. S3c-S3f). Collectively, these results showed that UBE2O promoted BC cell proliferation, EMT and CSPs through the AMPK $\alpha 2 /$ mTORC1 axis and that targeting UBE2O may be a promising strategy for $\mathrm{BC}$ therapy.

\section{MYC transcriptionally promoted UBE2O expression and exerted a positive feedback loop in $\mathrm{BC}$ cells}

As mentioned above, UBE2O was commonly overexpressed in $\mathrm{BC}$, so we investigated the regulatory mechanism of UBE2O in BC cells. The JASPAR database was used to identify potential UBE2O transcription factors. Coincidentally, we found that there were two potential binding sites for MYC in the UBE2O promoter region (Fig. 8a). In addition, we demonstrated that there was a significant linear correlation between UBE2O and MYC in BC patients (Fig. 4d). Therefore, siRNAs targeting MYC were transfected into MDA-MB-231 and MCF7 cells. The efficiency of MYC silencing was detected by western blotting (Fig. 8b) and qRT-PCR (Fig. S3g), as well as the relative UBE2O expression. The results revealed that UBE2O mRNA and protein expression levels (Fig. 8b, c) were decreased after silencing MYC in MDA-MB-231 and MCF-7 cells. Then, we constructed two sets of luciferase reporter plasmids (sequence 1 and sequence 2) containing the wild-type or mutant MYC-binding sites of the UBE2O promoter region. The data in Fig. $8 \mathrm{~d}$ revealed that the co-transfection of MYC and UBE2O-sequence 1 reporter plasmids in $293 \mathrm{~T}$ cells led to increased luciferase activity, and the rate of increase was positively correlated with the MYC plasmid dose. However, we failed to observe any differences in luciferase activity between the mutant groups. For sequence 2, the luciferase reporter assays showed that there were no significant differences in luciferase activity in the wild-type and mutant plasmid groups with increasing MYC plasmid doses. These results indicated that MYC could bind sequence 1 in the UBE2O promoter region and activate UBE2O transcription. Moreover, ChIP assays confirmed that MYC could directly bind to sequence 1 of the UBE2O promoterspecific region in both MDA-MB-231 and MCF-7 cells (Fig. 8e). Taken together, these results confirmed that MYC could activate the transcription of UBE2O, thus forming a positive feedback loop in BC cells.

\section{Discussion}

From the experiments above, we can draw the following conclusions: (1) UBE2O is highly expressed in BC tissues, and this high expression is related to poor prognosis in BC patients. (2) UBE2O promotes $\mathrm{BC}$ cell proliferation and EMT and maintains BC cell CSPs. (3) UBE2O promotes the malignant phenotype of $\mathrm{BC}$ cells by mediating AMPK $\alpha 2$ ubiquitination and targeting the UBE2O/ AMPK $\alpha 2 / m$ TORC1 axis. (4) As a downstream oncoprotein of UBE2O, MYC transcriptionally promotes UBE2O expression and exerts a positive feedback loop in $\mathrm{BC}$ cells.

As a large E2 ubiquitin-conjugation enzyme, UBE2O displays both E2 and E3 activities, and UBE2O deregulation is involved in various types of human cancers. UBE2O had been proved to decrease the stability of wildtype mixed lineage leukaemia (MLL) in a polyubiquitination-dependent manner, which may accelerate MLL cell proliferation and result in aggressive leukaemia $^{32}$. Vila et al. ${ }^{28}$ introduced UBE2O deletion into a series of transgenic mouse models of spontaneous cancer and revealed that UBE2O could facilitate tumour progression and metastasis in a UBE2O/AMPK $\alpha 2 /$ HIF-1adependent manner. However, the relationship between the UBE2O status of $\mathrm{BC}$ patients and their clinicopathological situation has not been fully reported. Our study demonstrated that UBE2O is overexpressed in BC tissues. $\mathrm{BC}$ patients with high UBE2O expression tend to have a high risk of tumour metastasis and poor prognosis. Moreover, we used a clinically relevant concentration of ATO to treat BC cells, and the results confirmed that ATO could significantly diminish the protumour ability of UBE2O. Thus, pharmacologically blocking UBE2O might be a promising target for $\mathrm{BC}$ treatment.

AMPK is an essential intracellular sensor of energy and metabolism, and its malfunction is associated with many types of human cancers. Gao et al. ${ }^{33}$ reported that the AMPK $\alpha 2$ subunit is mutated at a frequency of $0.2-10 \%$ 


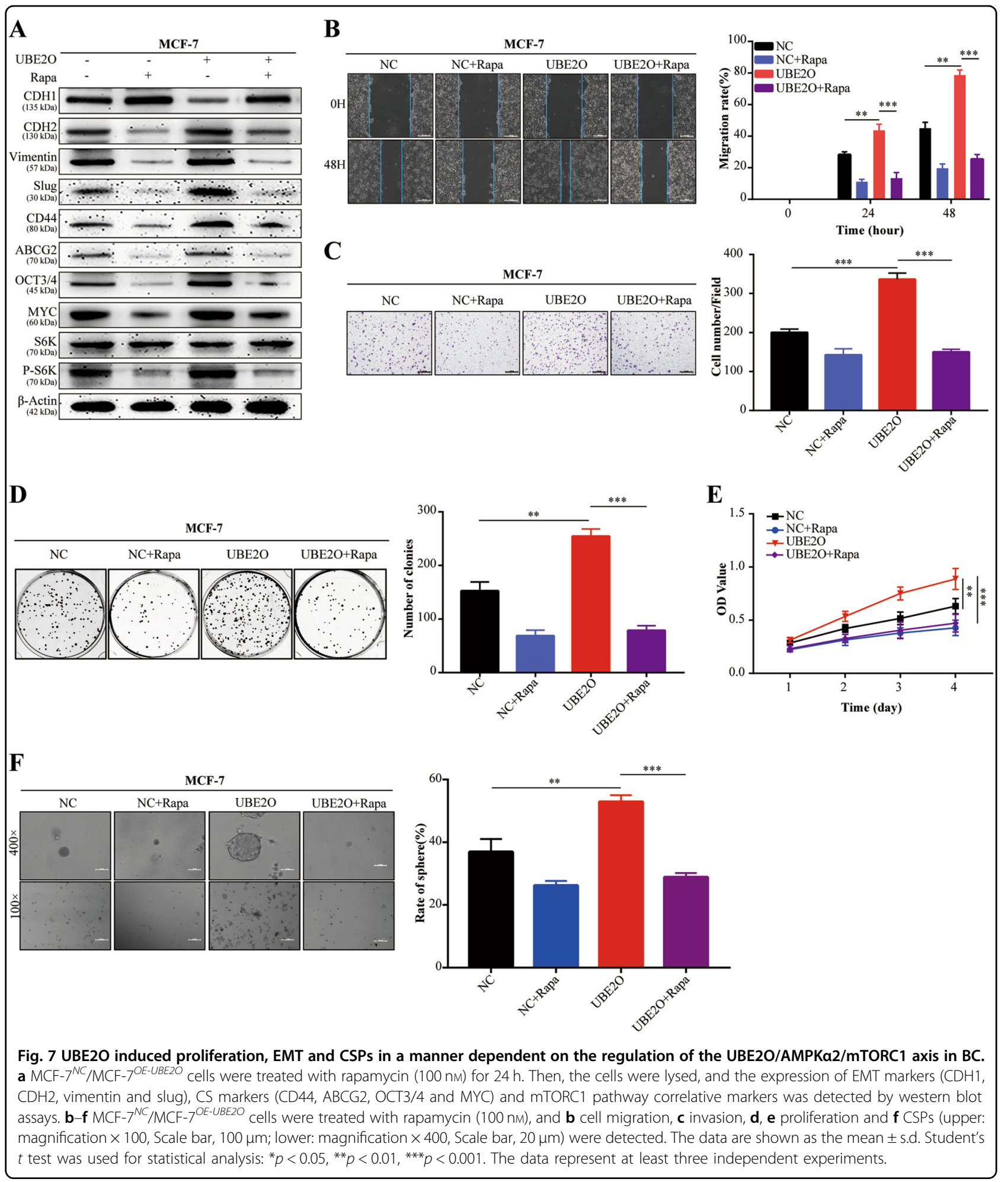

across all human cancers. AMPK $\alpha 2$ deregulation disrupts the tumour suppression capabilities of AMPK and then induces cancer cell progression. Our study confirmed that UBE2O promotes BC cell EMT and endows BC cell CSPs in an AMPK $\alpha 2$ ubiquitination-dependent manner. EMT plays a crucial role in tumour invasiveness and metastasis. During BC progression, EMT furnishes BC cells with selfrenewal capability and drives cancer stemness marker expression, contributing to the therapy resistance, immune escape and metastasis of $\mathrm{BC}$ cells ${ }^{34}$. In this study, 
A

Putative MYC binding sites in UBE2O sequence

\begin{tabular}{|l|l|l|l|l|l|l|}
\hline Name & Score & Relative score & Sequence ID & Start & End & $\begin{array}{l}\text { predicted site } \\
\text { sequence }\end{array}$ \\
\hline MYC & 10.1582 & 0.87992166005 & UBE2O & 846 & 857 & agccatgtgcca \\
\hline MYC & 7.19962 & 0.818154617249 & UBE2O & 943 & 954 & gacctcgtgatc \\
\hline
\end{tabular}

UBE2O promotor sequencel :

-ACTAC AGCCATGTGCCA CCACG -

UBE2O promotor sequence 2:
—CTCTT GACCTCGTGATC CGCCC -

C

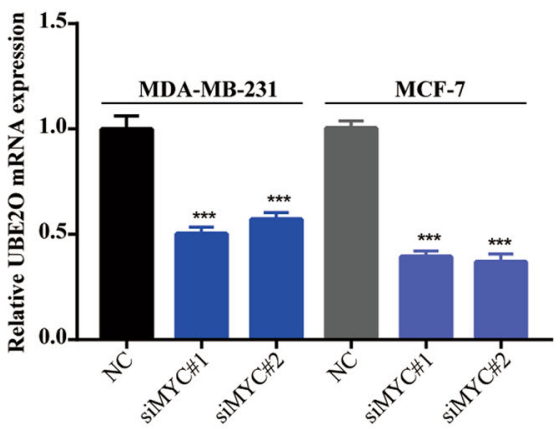

D

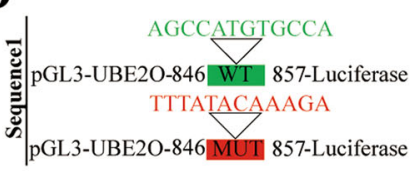

pGL3-UBE2O-846 MUT 857-Luciferase

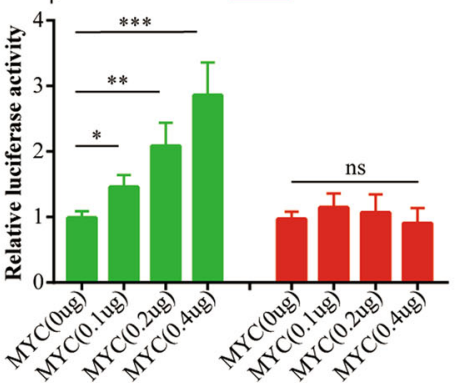

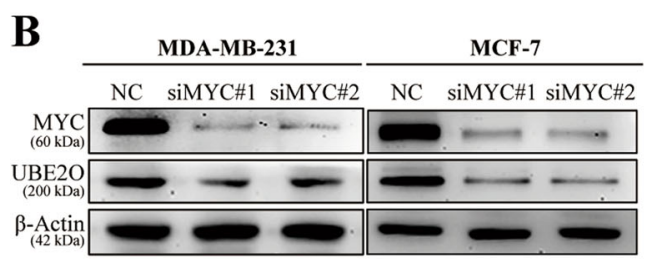

E

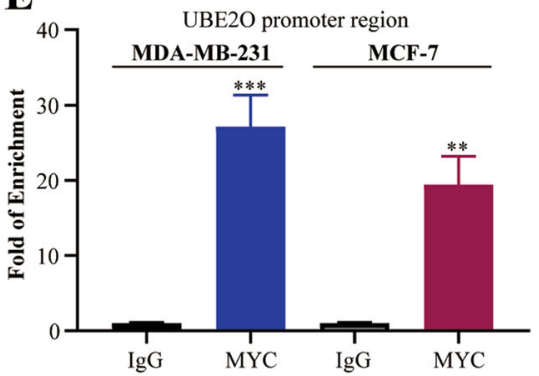

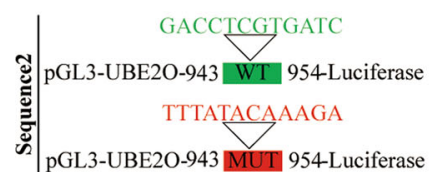

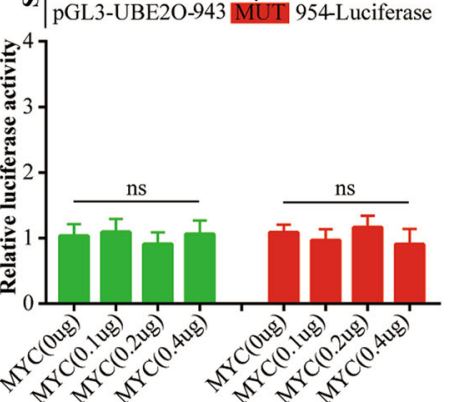

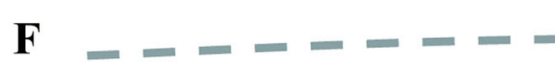
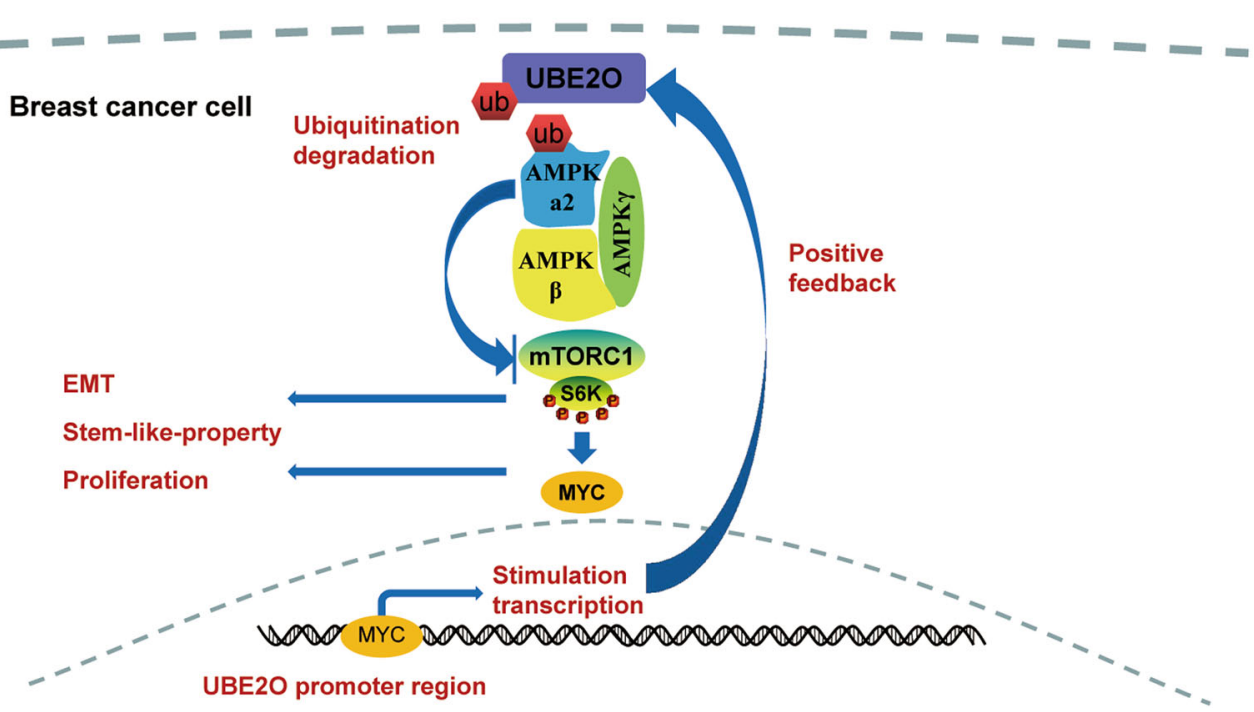

Fig. 8 (See legend on next page.) 
(see figure on previous page)

Fig. 8 MYC transcriptionally promoted UBE2O expression, and a positive feedback loop existed in BC cells. a Two putative MYC-binding sites in the UBE2O promoter region were forecasted by the JASPAR database. b, c siRNAs targeting MYC were transfected into MDA-MB-231 and MCF-7 cells. Expression changes in UBE2O were detected by qRT-PCR and western blot assays. $\mathbf{d}$ Wild-type or mutant UBE2O promoter reporter constructs targeting the two putative binding sites of MYC were separately transfected into the 293 T cell line. Various amounts of MYC plasmids were also transfected, and luciferase assays were performed. e ChIP assays were performed in the indicated BC cells with IgG or MYC antibodies. qRT-PCR was used to detect the fragments of the UBE2O promoter region. $\mathbf{f}$ Structure chart showing that the UBE2O/AMPKa2/mTORC1/MYC axis constituted a positive feedback loop in BC cells. The data are shown as the mean \pm s.d. Student's $t$ test was used for statistical analysis: ${ }^{*} p<0.05,{ }^{* *} p<0.01,{ }^{* * *} p<$ 0.001. The data represent at least three independent experiments.

AMPK $\alpha 2$ knockdown remarkably reverses the antiEMT

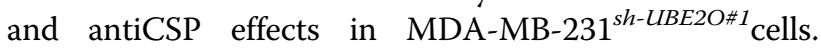
Treating MCF-7 cells with rapamycin disrupts the proEMT and prostemness properties established by overexpressing UBE2O. These results confirmed that UBE2O mediates $\mathrm{BC}$ cell biotumour behaviours in an AMPK $\alpha 2 / m$ TORC1-dependent manner. In addition, AMPK could inhibit cancer progression by activating $\mathrm{p} 53$, p27 and the antiWarburg effect ${ }^{35-37}$. There may also be other antitumour functions of AMPK that remain to be further investigated.

MYC is a well-characterised oncoprotein that is upregulated in $30-50 \%$ of $\mathrm{BC}$ patients. MYC contributes to BC cell progression and metastasis and induces CSPs in multiple tumour biological processes ${ }^{38}$. MYC acts as a transcriptional factor that regulates the transcription of over $15 \%$ of human genes, such as CCND, CDK4 and E2F1, which are involved in tumour progression ${ }^{39}$. In this study, we demonstrated that UBE2O upregulates MYC expression through the AMPK $\alpha 2 / \mathrm{mTORC} 1$ axis and that MYC could bind to the promoter region of UBE2O, thus transcriptionally promoting UBE2O expression. The selfcontrol positive feedback loop further highlights the significance of the UBE2O/AMPK $\alpha 2 / \mathrm{mTORC} 1-\mathrm{MYC}$ axis in $\mathrm{BC}$.

In conclusion, our study revealed that $\mathrm{UBE} 2 \mathrm{O}$ functions as a potential prognostic marker of $\mathrm{BC}$ and that the UBE2O/AMPK $\alpha 2 / m$ TORC1-MYC axis forms a positive feedback loop in BC cells, which promotes $\mathrm{BC}$ cell proliferation and EMT and endows BC cells with CSPs. Our research provides crucial theoretical evidence for the function of UBE2O in $\mathrm{BC}$.

\section{Acknowledgements}

This work was supported by funding from the National Natural Science Foundation of China (Grant Number 81372837) and the Scientific Research Project of Education Department of Heilongjiang province (Grant Number 1253HQ002).

\section{Conflict of interest}

The authors declare that they have no conflict of interest.

\section{Publisher's note}

Springer Nature remains neutral with regard to jurisdictional claims in published maps and institutional affiliations.
Supplementary Information accompanies this paper at (https://doi.org/ 10.1038/s41419-019-2194-9).

Received: 1 July 2019 Revised: 25 November 2019 Accepted: 3 December 2019

Published online: 06 January 2020

\section{References}

1. Berleth, E. S. \& Pickart, C. M. Mechanism of ubiquitin conjugating enzyme E2230K: catalysis involving a thiol relay? Biochemistry 35, 1664-1671 (1996).

2. Yokota, T. et al. Identification, tissue expression, and chromosomal position of a novel gene encoding human ubiquitin-conjugating enzyme E2-230k. Gene 267, 95-100 (2001).

3. Zhang, X. et al. Fine-tuning BMP7 signalling in adipogenesis by UBE2O/ E2-230K-mediated monoubiquitination of SMAD6. EMBO J. 32, 996-1007 (2013).

4. Zhang, X., Zhang, J., Zhang, L., van Dam, H. \& ten Dijke, P. UBE2O negatively regulates TRAF6-mediated NF-kappaB activation by inhibiting TRAF6 polyubiquitination. Cell Res. 23, 366-377 (2013).

5. Chen, S. et al. Ubiquitin-conjugating enzyme UBE2O regulates cellular clock function by promoting the degradation of the transcription factor BMAL1. J. Biol. Chem. 293, 11296-11309 (2018).

6. Lin, M. et al. DNA copy number gains in head and neck squamous cell carcinoma. Oncogene 25, 1424-1433 (2006).

7. Rice, K. L. et al. Analysis of genomic aberrations and gene expression profiling identifies novel lesions and pathways in myeloproliferative neoplasms. Blood Cancer J. 1, e40 (2011).

8. Toffoli, S. et al. Identification by array comparative genomic hybridization of a new amplicon on chromosome 17q highly recurrent in BRCA1 mutated triple negative breast cancer. Breast Cancer Res. 16, 466 (2014).

9. Davies, S. P. et al. Purification of the AMP-activated protein kinase on ATPgamma-sepharose and analysis of its subunit structure. Eur. J. Biochem. 223 351-357 (1994).

10. Munday, M. R., Campbell, D. G., Carling, D. \& Hardie, D. G. Identification by amino acid sequencing of three major regulatory phosphorylation sites on rat acetyl-CoA carboxylase. Eur. J. Biochem. 175, 331-338 (1988).

11. Dasgupta, B. \& Chhipa, R. R. Evolving lessons on the complex role of AMPK in normal physiology and cancer. Trends Pharmacol. Sci. 37, 192-206 (2016).

12. Hardie, D. G. AMP-activated protein kinase: maintaining energy homeostasis at the cellular and whole-body levels. Annu. Rev. Nutr. 34, 31-55 (2014).

13. Hawley, S. A. et al. Use of cells expressing gamma subunit variants to identify diverse mechanisms of AMPK activation. Cell Metab. 11, 554-565 (2010).

14. Hardie, D. G. AMP-activated protein kinase: an energy sensor that regulates all aspects of cell function. Genes Dev. 25, 1895-1908 (2011).

15. Kim, Y. H. et al. AMPKalpha modulation in cancer progression: multilayer integrative analysis of the whole transcriptome in Asian gastric cancer. Cancer Res. 72, 2512-2521 (2012).

16. Tong, W. H. et al. The glycolytic shift in fumarate-hydratase-deficient kidney cancer lowers AMPK levels, increases anabolic propensities and lowers cellular iron levels. Cancer Cell 20, 315-327 (2011).

17. Mo, J. S. et al. Cellular energy stress induces AMPK-mediated regulation of YAP and the Hippo pathway. Nat. Cell Biol. 17, 500-510 (2015).

18. Shaw, R. J. et al. The tumor suppressor LKB1 kinase directly activates AMPactivated kinase and regulates apoptosis in response to energy stress. Proc. Natl Acad. Sci. USA 101, 3329-3335 (2004). 
19. Hardie, D. G. \& Pan, D. A. Regulation of fatty acid synthesis and oxidation by the AMP-activated protein kinase. Biochem. Soc. Trans. 30, 1064-1070 (2002).

20. Inoki, K., Zhu, T. \& Guan, K. L. TSC2 mediates cellular energy response to control cell growth and survival. Cell 115, 577-590 (2003).

21. Gwinn, D. M. et al. AMPK phosphorylation of raptor mediates a metabolic checkpoint. Mol. Cell 30, 214-226 (2008).

22. Laderoute, K. R. et al. 5'-AMP-activated protein kinase (AMPK) is induced by low-oxygen and glucose deprivation conditions found in solid-tumor microenvironments. Mol. Cell Biol. 26, 5336-5347 (2006).

23. Rios, M. et al. Lipoprotein internalisation induced by oncogenic AMPK activation is essential to maintain glioblastoma cell growth. Eur. J. Cancer $\mathbf{5 0}$ 3187-3197 (2014).

24. Tennakoon, J. B. et al. Androgens regulate prostate cancer cell growth via an AMPK-PGC-1alpha-mediated metabolic switch. Oncogene 33, 5251-5261 (2014).

25. Bergeron, R. et al. Chronic activation of AMP kinase results in NRF-1 activation and mitochondrial biogenesis. Am. J. Physiol. Endocrinol. Metab. 281 E1340-E1346 (2001).

26. Ma, F. et al. Long non-coding RNA FGF13-AS1 inhibits glycolysis and stemness properties of breast cancer cells through FGF13-AS1/IGF2BPs/Myc feedback loop. Cancer Lett. 450, 63-75 (2019).

27. Vadakekolathu, J. et al. MTSS1 and SCAMP1 cooperate to prevent invasion in breast cancer. Cell Death Dis. 9, 344 (2018).

28. Vila, I. K. et al. A UBE2O-AMPKalpha2 axis that promotes tumor initiation and progression offers opportunities for therapy. Cancer Cell 31, 208-224 (2017).

29. Tang, J. et al. LncRNA DANCR upregulates PI3K/AKT signaling through activating serine phosphorylation of RXRA. Cell Death Dis. 9, 1167 (2018).
30. Zhang, J. et al. Guanylate-binding protein 2 regulates Drp1-mediated mitochondrial fission to suppress breast cancer cell invasion. Cell Death Dis. 8, e3151 (2017).

31. Hu, J., Li, G., Zhang, P., Zhuang, X. \& Hu, G. A CD44v(+) subpopulation of breast cancer stem-like cells with enhanced lung metastasis capacity. Cell Death Dis. 8, e2679 (2017).

32. Liang, $\mathrm{K}$. et al. Therapeutic targeting of MLL degradation pathways in MLLrearranged leukemia. Cell 168, 59-72 e13 (2017).

33. Gao, J. et al. Integrative analysis of complex cancer genomics and clinical profiles using the cBioPortal. Sci. Signal. 6, pl1 (2013).

34. Wang, M. H. et al. Epithelial cell adhesion molecule overexpression regulates epithelial-mesenchymal transition, stemness and metastasis of nasopharyngeal carcinoma cells via the PTEN/AKT/mTOR pathway. Cell Death Dis. 9, 2 (2018).

35. Vazquez-Martin, A. et al. Activation of AMP-activated protein kinase (AMPK) provides a metabolic barrier to reprogramming somatic cells into stem cells. Cell Cycle 11, 974-989 (2012)

36. Faubert, B. et al. AMPK is a negative regulator of the Warburg effect and suppresses tumor growth in vivo. Cell Metab. 17, 113-124 (2013).

37. Luo, Z., Zang, M. \& Guo, W. AMPK as a metabolic tumor suppressor: control of metabolism and cell growth. Future Oncol. 6, 457-470 (2010).

38. Blancato, J., Singh, B., Liu, A., Liao, D. J. \& Dickson, R. B. Correlation of amplification and overexpression of the c-myc oncogene in high-grade breast cancer: FISH, in situ hybridisation and immunohistochemical analyses. Br. J. Cancer 90, 1612-1619 (2004).

39. Meyer, N. \& Penn, L. Z. Reflecting on 25 years with MYC. Nat. Rev. Cancer 8 976-990 (2008) 